05

\title{
Распространение спиновых волн в микроструктурах на основе пленок железоиттриевого граната, декорированных ферромагнитным металлом
}

\author{
(C) Ю.В. Хивинцев ${ }^{1,2}$, Г.М. Дудко ${ }^{1}$, В.К. Сахаров ${ }^{1}$, Ю.В. Никулин ${ }^{1,2}$, Ю.А. Филимонов ${ }^{1,2}$ \\ ${ }^{1}$ Саратовский филиал Института радиотехники и электроники им. В.А. Котельникова РАН, \\ Саратов, Россия \\ ${ }^{2}$ Саратовский государственный университет им. Н.Г. Чернышевского, \\ Саратов, Россия \\ E-mail: khivintsev@gmail.com
}

Поступила в Редакцию 15 апреля 2019 г.

В окончательной редакции 22 апреля 2019 г.

Принята к публикации 24 апреля 2019 г.

\begin{abstract}
Экспериментально и теоретически исследовано распространение спиновых волн в пленке железоиттриевого граната, декорированной микроструктурами из никелевой пленки. Показано, что выбором геометрии микроструктур никеля можно управлять затуханием и формировать волновые пучки спиновых волн.
\end{abstract}

Ключевые слова: спиновая волна, пленка железоиттриевого граната, декорирование ферромагнитным металлом, волновые каналы.

DOI: $10.21883 /$ FTT.2019.09.48108.15N

\section{1. Введение}

Одним из активно развиваемых направлений современной микроэлектроники является магноника, в основе которой лежит использование для обработки информации эффектов распространения спиновых волн (CB) в магнитных микро- и наноструктурах [1-3]. При этом важным звеном на пути построения миниатюрных магнонных устройств с развитой архитектурой является задача формирования и управления потоками СВ. В частности, формирование отдельных потоков СВ и обеспечение их взаимодействия необходимо для построения магнонной логики и устройств голографической памяти, основанных на интерференции СВ [4-9]. Представляет интерес также осуществление пространственночастотного разделения СВ и создание на их основе многоканальных СВЧ фильтров [10].

Применительно к тонкопленочным структурам можно выделить два основных способа разделения потоков СВ. Первый основан на анизотропии свойств СВ и применении возбуждающих систем сложной формы [10-16] для осуществления эффектов фокусировки и пространственного разделения потоков СВ в тонкопленочном волноводе. Второй способ предполагает локализацию потока СВ за счет использования тонкопленочных волноводов ограниченной ширины [4-9,17-20].

В последнем случае, как правило, применяются волноводы и системы на их основе, сформированные путем геометрического структурирования магнитной пленки литографическими методами. В качестве магнитной среды для формирования таких волноводов в экспериментах зачастую используется железоиттриевый гранат (ЖИГ), как материал, характеризующийся рекордно низким за- туханием СВ среди известных магнетиков. При этом имеются определенные технологические сложности с травлением ЖИГ (наличие бокового подтрава при химическом травлении и невысокая разрешающая способность лазерного скрайбирования делают их малопригодными для формирования структур из ЖИГ с латеральными размерами $<50 \mu \mathrm{m}$, а относительно низкая скорость ионного травления затрудняет формирование структур из пленок ЖИГ толщиной $>1 \mu \mathrm{m})$. Поэтому актуальным является поиск и изучение иных механизмов формирования потоков СВ ограниченной ширины, не требующих структурирования пленки ЖИГ. Отметим также, что ранее рассматривалась возможность формирования волновых пучков за счет создания неоднородных полей подмагничивания определенной формы [21]. Однако опять же такой подход сопряжен с существенными техническими трудностями в реализации.

В нашей работе исследуется возможность использования для этих целей декорирования поверхности ЖИГ ферромагнитным металлом - никелем (Ni). Такие структуры более просты в изготовлении (за счет более развитых и менее затратных технологий обработки $\mathrm{Ni}$ по сравнению с ЖИГ), и в то же время обеспечивают два источника влияния на свойства СВ в ЖИГ: через взаимодействие с электронами проводимости [22] и через взаимодействие с магнитной системой $\mathrm{Ni.}$ При этом отметим, что ранее [23] исследовалась возможность использования проводящих и магнитных материалов в качестве поглотителей СВ для борьбы с паразитными отражениями в тонкопленочных образцах ЖИГ. Достаточно подробно были изучены особенности спектра СВ в двуслойных магнитных структуpax, см. например [24]. Теоретически рассматривалась 
также возможность формирования волновых каналов для СВ за счет декоририрования немагнитным металлом [25]. Однако, возможность формирования волновых пучков с помощью декорирования ферромагнитным металлом, насколько известно авторам, ранее не обсуждалась.

\section{2. Исследуемые структуры}

На рис. 1 показаны исследуемые структуры на основе пленки ЖИГ, декорированной пленкой $\mathrm{Ni}$, с микроантеннами (МА) для возбуждения-приема СВ, сформированными на поверхности ЖИГ. МА представляли собой микрополоски шириной $w_{\mathrm{MA}}=8 \mu \mathrm{m}$ и длиной $50 \mu \mathrm{m}$ с квадратными контактными площадками на обоих концах размерами $100 \times 100 \mu \mathrm{m}$. МА 1 использовалась в качестве возбуждающей, МА 2 и 3 - приемных. Расстояние $L_{\mathrm{MA}}$ между МА 1 и 2 составляло $476 \mu \mathrm{m}$, между МА 2 и $3-350 \mu \mathrm{m}$.

Структура А была свободна от Ni. В структуре В между возбуждающей и приемными МА симметрично по центру располагалась полоска $\mathrm{Ni}$ шириной $L_{\mathrm{Ni}}=400 \mu \mathrm{m}$. Структура С отличалась от структуры В наличием микроканала шириной $50 \mu \mathrm{m}$ в полоске $\mathrm{Ni}$ между МА 1 и 2. В структуре $\mathrm{D}$ из пленки $\mathrm{Ni}$ была сформирована микрополоска шириной $50 \mu \mathrm{m}$ между МА 1 и 2, то есть данная структура была обращенной по отношению к С. В структуре Е микроканал в пленке Ni был сформирован между МА 1 и 3.

Отметим, что все структуры были симметричны относительно оси, проходящей через центры МА 1 и 2. То есть справа от МА 2 располагалась еще одна МА,

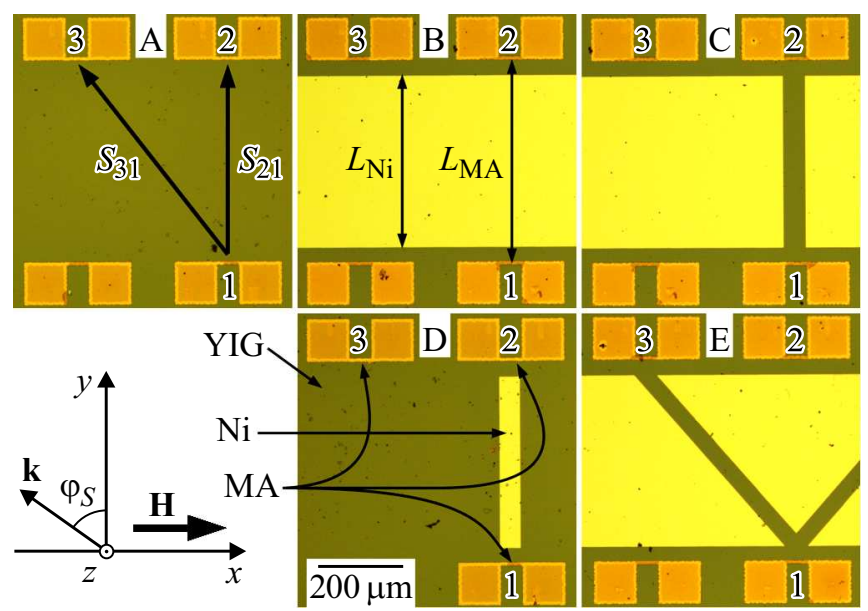

Pис. 1. Фотографии экспериментальных структур А-E (см. соответствующие подписи вверху фотографий). Различные цвета на фотографиях соответствуют различным материалам: ЖИГ, Ni и медным МА с контактными площадками (см. стрелки с соответствующими пояснениями на фотографии структуры D). Цифрами указаны номера MA на каждой структуре. симметричная 3, и в структуре Е имелся соответствующий канал к этой антенне. Однако результаты, полученные для этой дополнительной МА, для всех типов структур были аналогичны результатам для МА 3, и поэтому далее не обсуждаются.

Для изготовления структур использовалась пленка ЖИГ толщиной $d_{\text {YIG }}=3.5 \mu \mathrm{m}$ с намагниченностью $4 \pi M_{\mathrm{YIG}}=1750 \mathrm{G}$ и полушириной линии ферромагнитного резонанса $\Delta H=0.5 \mathrm{Oe}$, выращенная жидкофазной эпитаксией на подложке гадолиний галлиевого граната. Все структуры (A-E) изготавливались на одной подложке в рамках единой процедуры. Сначала с помощью технологий магнетронного распыления, фотолитографии и ионного травления на поверхности пленки ЖИГ формировались необходимые микроструктуры из $\mathrm{Ni}$ толщиной $d_{\mathrm{Ni}}=0.35 \mu \mathrm{m}$. Затем с использованием тех же технологий изготавливались МА с контактными площадками из меди такой же толщины. При изготовлении МА, на этапе фотолитографии, осуществлялось совмещение топологии микроантенн с микроструктурами из $\mathrm{Ni}$ с точностью не хуже $2 \mu \mathrm{m}$.

\section{3. Методика измерений}

Тестирование структур проводилось с помощью векторного анализатора цепей и СВЧ зондовой станции в интервале частот 1-7 GHz. Измерялись амплитудночастотные характеристики (АЧХ) и фазо-частотные характеристики (ФЧХ) коэффициентов передачи между MA 1 и МА 2, 3 ( $S_{21}$ и $S_{31}$ соответственно) при различной величине поля подмагничивания $H=120-1322 \mathrm{Oe}$, приложенного вдоль оси $x$ (см. рис. 1). ФЧХ использовались для расчета экспериментальной дисперсии СВ согласно выражению

$$
k=-\left[\operatorname{Arg}\left(S_{21}^{\mathrm{X}}(f)\right)-\operatorname{Arg}\left(S_{21}^{\mathrm{A}}(f)\right) \cdot\left(1-L_{\mathrm{Ni}} / L_{\mathrm{MA}}\right)\right] / L_{\mathrm{Ni}},
$$

где $k$ и $f-$ волновое число и частота СВ соответственно, $\mathrm{X}$ - тип структуры $(\mathrm{X}=\mathrm{A}-\mathrm{D})$. Отметим, что данное выражение учитывает тот факт, что в структурах, декорированных никелем, СВ часть пути $\left(L_{\mathrm{MA}}-L_{\mathrm{Ni}}\right)$ распространяется по свободной пленке, и для таких структур описывает дисперсию именно на декорированном участке пути $\left(L_{\mathrm{Ni}}\right)$.

Мощность сигнала, подаваемого на МА 1, составляла $-40 \mathrm{dBm}$, что ниже порога параметрической неустойчивости СВ. Для повышения чувствительности измерений при столь малой мощности сигнал с выхода исследуемой структуры перед подачей на анализатор цепей пропускался через малошумящий предусилитель с коэффициентом усиления $+25 \mathrm{~dB}$. Перед проведением измерений выполнялась калибровка, позволяющая исключить влияние кабелей, зондов и усилителя на результаты измерений.

Для снижения влияния уровня прямой электромагнитной наводки между МА, результаты измерений подверга- 
лись дополнительной математической обработке: из характеристик, полученных при выбранном $H$, вычитались характеристики, отвечающие электромагнитной наводке, измеренные при $H=2.6 \mathrm{kOe}$, когда дно спектра $\mathrm{CB}$ $f_{\mathrm{H}}=\gamma \cdot H$, где $\gamma=2.8 \mathrm{MHz} / \mathrm{Oe}-$ гиромагнитное отношение для ЖИГ, выше $7 \mathrm{GHz}$.

\section{4. Методика расчета}

Выбранная геометрия подмагничивания отвечает распространению вдоль оси $y$ (см. рис. 1) поверхностной магнитостатической волны (ПМСВ). При этом на данный момент не существует теории, которая бы описывала динамические процессы, связанные с распространением ПМСВ в слоистой структуре из двух магнетиков, один из которых является проводником. Поэтому для анализа экспериментальных результатов мы использовали известные теории по влиянию на дисперсию и затухание ПМСВ металлизации ЖИГ [22,26] и наличия дополнительного магнитного слоя [24]. Для анализа привлекалось также микромагнитное моделирование, которое учитывало динамические процессы как в ЖИГ, так и в $\mathrm{Ni}$, но не учитывало электрических свойств $\mathrm{Ni}$.

Расчет дисперсии и пространственного декремента $k^{\prime \prime}$ ПМСВ, распространяющейся вдоль $y$, в свободной пленке ЖИГ $\left(k_{\mathrm{YIG}}^{\prime \prime}\right)$ и нагруженной металлом конечной проводимости $\left(k_{\mathrm{YIG}-\mathrm{Ni}}^{\prime \prime}\right)$ проводился с использованием дисперсионного соотношения, полученного в [26]. При этом удельное электрическое сопротивление металла $\rho$ и его относительная магнитная проницаемость $\mu$ выбирались соответствующими Ni: $\rho=7 \cdot 10^{-8} \Omega \cdot \mathrm{m}, \mu=100$. Геометрические и магнитные параметры пленки ЖИГ соответствовали эксперименту. Для сравнения с экспериментальными АЧХ полученные значения $k^{\prime \prime}$ пересчитывались в коэффициент затухания в логарифмическом масштабе $\Delta$ согласно выражениям

$$
\begin{aligned}
& \Delta=8.68 \cdot k_{\mathrm{YIG}}^{\prime \prime} \cdot L_{\mathrm{MA}} \text { для структуры } \mathrm{A} \text {, } \\
& \Delta=8.68 \cdot k_{\mathrm{YIG}}^{\prime \prime} \cdot\left(L_{\mathrm{MA}}-L_{\mathrm{Ni}}\right) \\
& +8.68 \cdot k_{\mathrm{YIG}-\mathrm{Ni}}^{\prime \prime} \cdot L_{\mathrm{Ni}} \quad \text { для структуры В. }
\end{aligned}
$$

Для расчета дисперсионной зависимости в двухслойной структуре из двух магнетиков использовались результаты [24]. При этом пренебрегалось затуханием спиновых волн. Геометрические и магнитные параметры пленки ЖИГ в расчетах соответствовали эксперименту. Намагниченность $\mathrm{Ni}$ принималась равной $4 \pi M_{\mathrm{Ni}}=6160 \mathrm{G}$.

Микромагнитное моделирование проводилось с помощью пакета программ OOMMF 1.2b0 [27]. При этом использовались характерная для ЖИГ и $\mathrm{Ni}$ обменная жесткость $A_{\mathrm{YIG}}=4 \cdot 10^{-7} \mathrm{erg} / \mathrm{cm}$ и $A_{\mathrm{Ni}}=9 \cdot 10^{-7} \mathrm{erg} / \mathrm{cm}$, а также характерный для этих материалов параметр затухания Гилберта $\alpha_{\mathrm{YIG}}=10^{-3}$ и $\alpha_{\mathrm{Ni}}=0.12$. Прочие параметры были такими же, как и в расчетах, описанных выше. При этом отметим, что все параметры, использовавшиеся в настоящей работе для расчетов, являются типичными для ЖИГ и Ni [28]. Размеры расчетной области по координатам $x, y$ и $z$ составляли 1350,570 и $3.85 \mu \mathrm{m}$ соответственно. Размеры ячеек были $d x=d y=3 \mu \mathrm{m}$ и $d z=0.35 \mu \mathrm{m}$. Отметим, что при выбранном $d z$ по толщине $\mathrm{Ni}$ укладывается одна ячейка, а по толщине ЖИГ - 10, что позволяет моделировать колебания намагниченности неоднородные по толщине ЖИГ.

Сначала рассчитывалось равновесное состояние намагниченности при выбранном стационарном поле подмагничивания $H$, ориентированном вдоль $x$. Затем к полю $H$ добавлялось поле $h_{\sim}=0.1 \sin (2 \pi f t)$ Ое, поляризованное вдоль оси $z$ и локализованное на участке $50 \times 8 \times 3.85 \mu \mathrm{m}$. Таким образом, осуществлялась поперечная накачка гармоническим сигналом на частоте $f$ в области, отвечающей МА. Амплитуда высокочастотного поля $h_{\sim}$ выбиралась достаточно малой $(0.1 \mathrm{Oe})$, чтобы избежать развития параметрической неустойчивости СВ и проявления связанных с этим эффектов.

В условиях такого внешнего воздействия осуществлялся расчет динамики намагниченности, и записывались мгновенные распределения одной из поперечных компонент намагниченности $-M_{z}$, характеризующей прецессию спинов по образцу, с шагом по времени $\Delta t=10^{-11} \mathrm{~s}$. Для того чтобы вычленить высокочастотную составляющую $M_{z}$ и построить карты распределения колебаний $M_{z}$ по образцу, из полученных данных вычиталось равновесное распределение $M_{z}$, рассчитанное при $h_{\sim}=0$.

Отметим, что размеры ячеек в микромагнитном моделировании значительно превышали обменную длину для ЖИГ и Ni и выбирались такими исходя из необходимости провести расчет для области, отвечающей экспериментальным образцам, при имеющейся вычислительной мощности. В этом случае, как известно [29], большой размер ячейки может вносить существенные искажения в динамические явления, определяющую роль для которых имеет обменное взаимодействие, и, в то же время, слабо влияет на длинноволновые возбуждения. В настоящей работе рассматриваются достаточно длинные $\mathrm{CB}\left(k<10^{4} \mathrm{rad} / \mathrm{cm}\right)$, вклад обменного взаимодействия в формирование которых достаточно мал, что позволяет рассчитывать на адекватность результатов моделирования эксперименту.

\section{5. Результаты и обсуждение}

Как известно [30], в касательно намагниченной свободной пленке ЖИГ могут распространяться два типа дипольных СВ: ПМСВ и обратная объемная магнитостатическая волна (ООМСВ), имеющие общую длинноволновую границу $f_{0}=\gamma \cdot\left(H\left(H+4 \pi M_{\text {YIG }}\right)\right)^{1 / 2}$. Спектр ПМСВ располагается выше $f_{0}$, а ООМСВ ниже. Групповая скорость низшей моды ООМСВ максимальна при распространении вдоль $H$ и уменьша- 

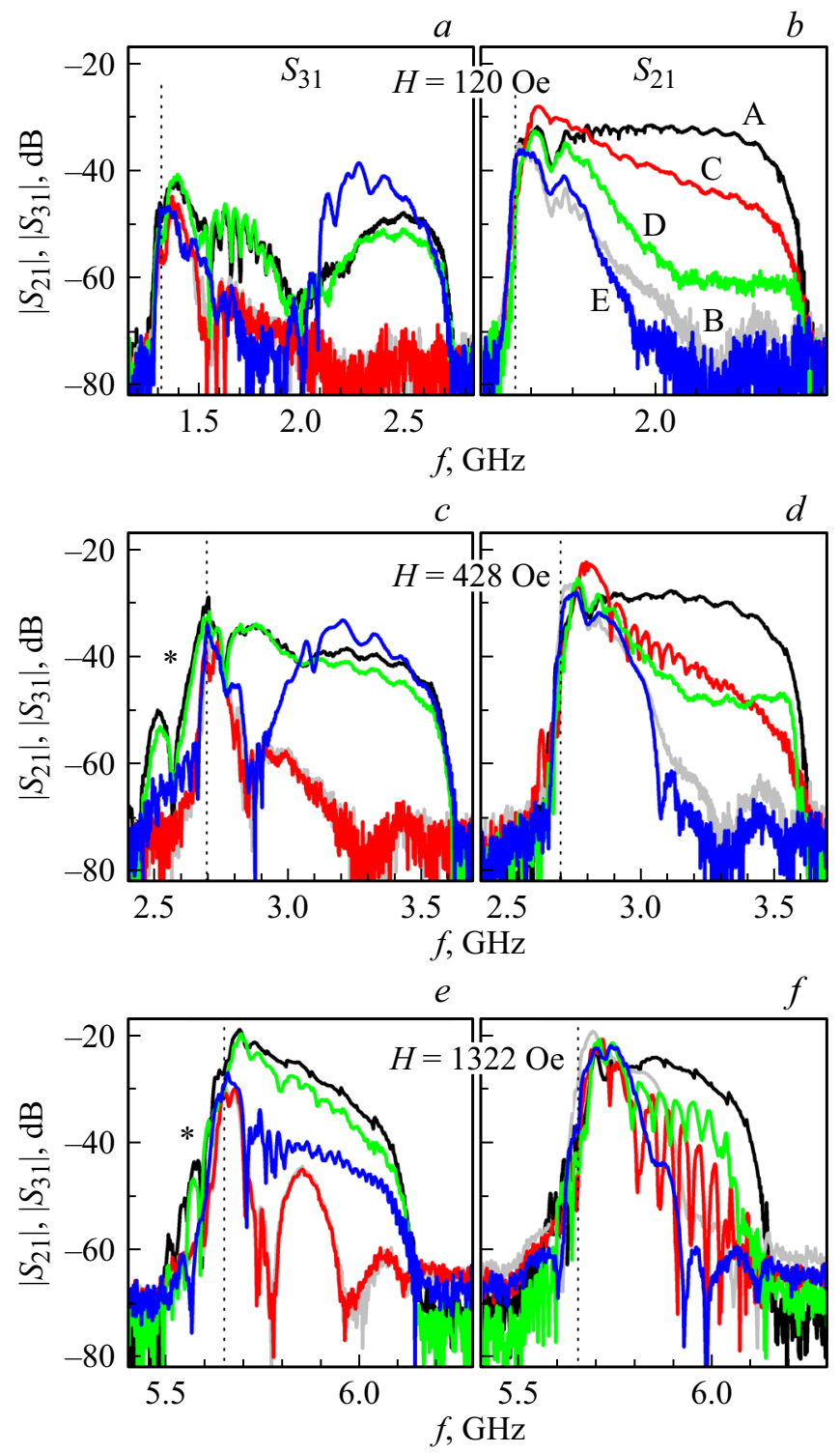

Рис. 2. АЧХ $S_{31}(a, c, e)$ и $S_{21}(b, d, f)$ для различных структур (см. обозначения кривых на $b$ ) при $H=120(a, b), 428(c, d)$ и 1322 Ое $(e, f)$. Вертикальный пунктир - положение $f_{0}$.

ется до нуля при развороте направления распространения на 90․ Групповая скорость ПМСВ, наоборот, максимальна при распространении перпендикулярно $H$ и уменьшается при отклонении от этого направления. При этом для ПМСВ имеется угол отсечки $\varphi_{S}=\operatorname{arctg}\left(\left(4 \pi M_{\text {YIG }} / H\right)^{1 / 2}\right)-$ см. рис. 1, больше которого ПМСВ не существует.

Для экспериментальных структур угол распространения между МА 1 и 3 относительно оси у составлял $\varphi_{13} \approx 36^{\circ}$, и условие распространения ПМСВ к MA $3\left(\varphi_{S}>\varphi_{13}\right)$ выполнялось до $H \approx 3200$ Ое, что значительно превышает величины полей в эксперименте. То есть для структуры А во всем интервале экспериментальных $H$ было возможно распространение ПМСВ с волновым вектором $k$, направленным как к МА 2, так и к МА 3. При этом возможность возбуждения волн МА 1 с волновыми векторами отличными от направления оси $y$ обусловлена конечной и довольно малой апертурой МА.

Действительно, в эксперименте для структуры А наблюдалось эффективное прохождение сигнала выше $f_{0}$ для обоих этих направлений распространения см. рис. 2. При этом низкочастотная граница зоны прохождения в $S_{21}$, как для структуры А, так и для всех остальных структур, хорошо совпадает с $f_{0}$ (небольшое несоответствие может быть отнесено на наличие анизотропии, которая не учитывалась при расчете $f_{0}$, а также на некоторое несоответствие экспериментальных параметров расчетным). Это свидетельствует о том, что передача сигнала в этом случае $\left(f>f_{0}\right)$ осуществляется исключительно ПМСВ.

В $S_{31}$ для структур A и D наблюдалась также дополнительная зона прохождения сигнала ниже $f_{0}$ (отмечена * на рис. 2, с и $e$ ), которая становилась заметной при $H>200$ Ое. Эту зону естественно связать с ООМСВ. При этом увеличение эффективности прохождения ООМСВ с ростом поля можно объяснить соответствующим увеличением групповой скорости для нее.

\section{Структура A}

Экспериментальная дисперсия ПМСВ для структуры А, полученная из $Ф Ч Х ~ S_{21}$, находилась в хорошем согласии с теоретической для свободной пленки ЖИГ -

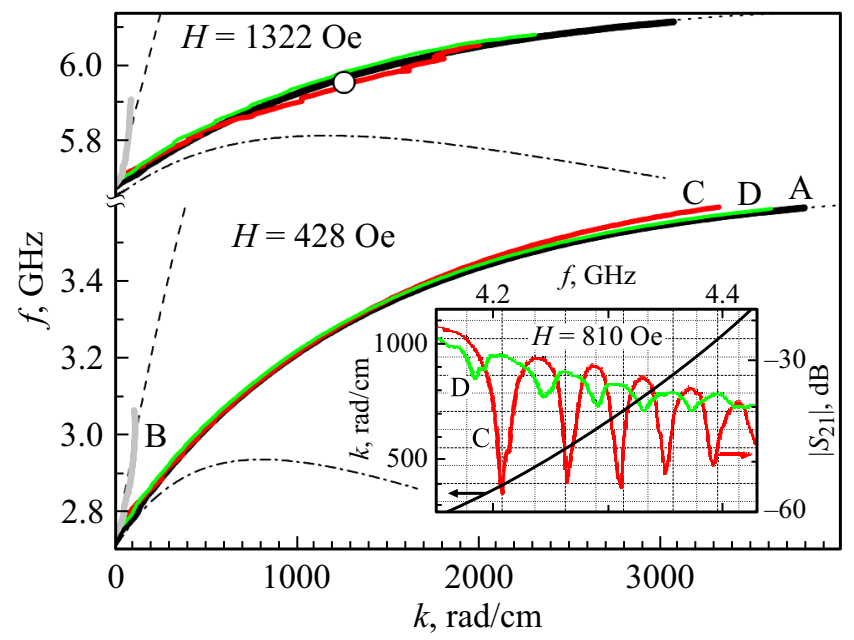

Рис. 3. Экспериментальная дисперсионная характеристика, полученная из $S_{21}$, для различных структур (см. обозначения кривых) при $H=428$ и 1322 Ое. Тонкая пунктирная линия теоретическая дисперсионная характеристика ПМСВ в свободной пленке ЖИГ. Штриховая и штрих-пунктирная линии расчет для структуры ЖИГ-Ni, где учтена проводимость $\mathrm{Ni}$ и его динамические магнитные свойства, соответственно. Кружок - данные микромагнитного моделирования для структуры А. На вставке - дисперсионная характеристика ПМСВ в свободной пленке ЖИГ и участок АЧХ $S_{21}$ для структур C и D при $H=810$ Oe. 

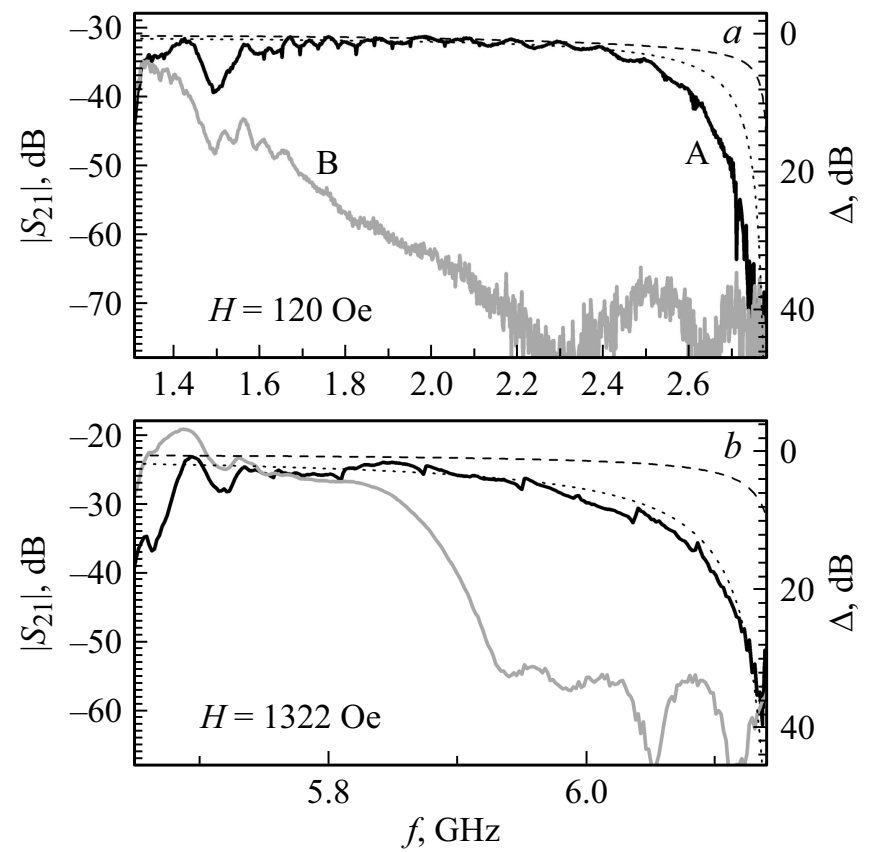

Рис. 4. Экспериментальные АЧХ $S_{21}$ для структур А и В (черная и серая сплошные линии соответственно), а также рассчитанные частотные зависимости затухания ПМСВ $\Delta$ для свободной пленки ЖИГ (пунктир), и нагруженной пленки Ni (штриховая линия) с учетом его проводимости при $H=120(a)$ и $1322 \mathrm{Oe}(b)$.

см. рис. 3. Максимальные $k$ в эксперименте составили $\sim 4 \cdot 10^{4} \mathrm{rad} / \mathrm{cm}$. Такое ограничение объясняется как резким падением эффективности возбуждения при

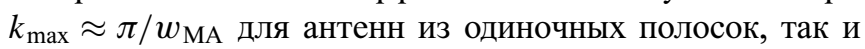
резким снижением групповой скорости коротких ПМСВ, сопровождающимся увеличением затухания.

Рассчитанные частотные зависимости затухания ПМСВ в свободной пленке ЖИГ соответствовали экспериментальным АЧХ $S_{21}$ для структуры А (см. рис. 4), если принять, что имеются дополнительные потери сигнала величиной $\Delta_{0}$ и соответственно сдвинуть кривые $\Delta$ относительно экспериментальных АЧХ на эту величину. При этом $\Delta_{0}$ составляла $\sim 31 \mathrm{~dB}$ для $H=120 \mathrm{Oe}$ и уменьшалась с ростом поля до $\sim 23 \mathrm{~dB}$ для $H=1322$ Ое. Отметим, что эти дополнительные потери связаны как с потерями на преобразование электромагнитных колебаний в колебания намагниченности, так и с тем, что МА 2 принимает лишь часть потока ПМСВ, формируемого MA 1 (см. рис. 5,a).

Для больших $H$ наблюдалось хорошее количественное соответствие частотных зависимостей $\Delta$ и $\left|S_{21}\right|$ (рис. $4, b)$, тогда как при малых $H$ наблюдалось расхождение в высокочастотной части (рис. 4,a). Такое расхождение можно объяснить тем, что в эксперименте при малых $H$ высокочастотная часть АЧХ формировалась за счет более коротких волн (см. рис. 3), с $k$ близкими к $k_{\max }$, и соответственно в этом случае более существенное влияние на формирование $\left|S_{21}\right|$ оказывает зависимость эффективности МА от $k$.

Отметим, что, согласно [30], можно было бы ожидать снижения высокочастотной границы спектра ПМСВ $f_{S}$ при распространении под углом к оси $y$ (от МА 1 к MA 3). Причем, чем выше $H$, тем большего снижения $f_{S}$ следует ожидать. В частности, для $H=1322$ Ое при распространении ПМСВ вдоль у теория [30] дает $f_{S} \approx 6.15 \mathrm{GHz}$, а при распространении под углом $\varphi_{13}-$ $f_{S} \approx 5.76 \mathrm{GHz}$. Однако в эксперименте высокочастотные границы АЧХ $S_{21}$ и $S_{31}$ совпадают во всем интервале $H$. Это связано с сильной анизотропией свойств ПМСВ, проявляющейся в различии направлений груп-

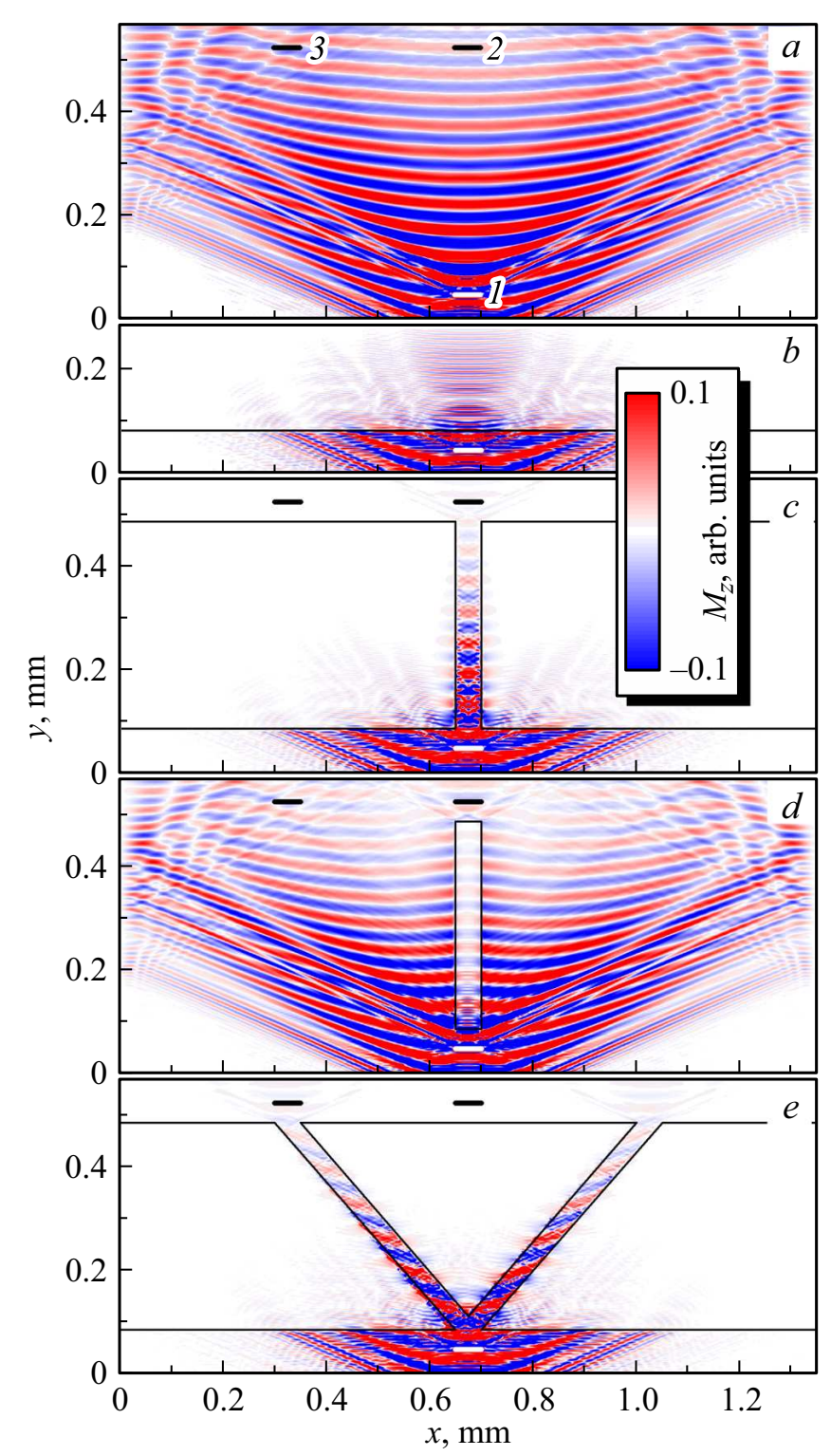

Рис. 5. Результаты микромагнитного моделирования: мгновенное распределение высокочастотной составляющей $M_{z}$ в структурах A-E $(a-e$ соответственно) при $H=1322 \mathrm{Oe}$ и $f=5.95 \mathrm{GHz}$. Жирные горизонтальные линии показывают положение МА, тонкие линии — границы пленки $\mathrm{Ni.}$ 
повой и фазовой скоростей [13]. Микромагнитное моделирование показывает (рис. 5,a), что МА 1 формирует ПМСВ, которая независимо от направления потока энергии в пределах местоположения МА 2 и 3 имеет направление фазовой скорости, близкое к оси $y$. Это и обусловливает равенство $f_{S}$ для различных направлений распространения сигнала, поскольку именно направление фазовой скорости в [30] определяет $f_{S}$.

Отметим также, что микромагнитное моделирование для структуры А дало $k$ близкое к экспериментальному - см. рис. 3. Это свидетельствует об адекватности моделирования экспериментальной ситуации.

\section{Структура В}

Для структуры В в эксперименте наблюдалось сильное подавление высокочастотной части спектра ПМСВ по сравнению со структурой А. Дисперсия для этой структуры, полученная из экспериментальных данных для участка прохождения, была близка к расчету, учитывающему электропроводность $\mathrm{Ni}$ (рис. 3). При этом следует отметить, что область прохождения сигнала ограничивалась длинами волн $\lambda>L_{\mathrm{Ni}}$.

Рассчитанные с учетом проводимости $\mathrm{Ni}$ частотные зависимости $\Delta$ для этого случая сильно отличались от экспериментальных АЧХ $S_{21}$ (рис. 4). Исходя из расчетов, влияние $\mathrm{Ni}$, как проводника, должно не увеличивать, а уменьшать затухание ПМСВ. Таким образом, электропроводность $\mathrm{Ni}$ не может объяснить сильного подавления ПМСВ в эксперименте.

Между тем, известно [24], что наличие дополнительного магнитного слоя, обладающего большей намагниченностью, может существенно перестраивать дисперсию ПМСВ в ЖИГ, снижая ее высокочастотную границу по сравнению со свободной пленкой. Для иллюстрации этого на рис. 3 приведены результаты расчета дисперсии ПМСВ в пленке ЖИГ, нагруженной магнитным слоем с параметрами, отвечающими $\mathrm{Ni}$. В эксперименте прохождение ПМСВ в структуре В наблюдается в более широком интервале частот, чем предсказывает теория. Однако это может быть обусловлено тем, что данная теория не учитывает проводимости $\mathrm{Ni}$. Таким образом, можно предположить, что наблюдаемое сужение зоны прохождения ПМСВ в структуре В по сравнению со случаем свободной пленки является отражением перестройки дисперсии за счет наличия магнитных свойств у Ni. Отметим также, что Ni является сильно диссипативной магнитной средой и может выступать в роли эффективного демпфера для магнитных колебаний в ЖИГ, и это также может являться одной из причин сильного подавления ПМСВ в структуре $\mathrm{B}$.

Микромагнитное моделирование для структуры В (рис. $5, b)$, также как и эксперимент, демонстрировало сильное подавление ПМСВ в высокочастотной части спектра. При этом микромагнитное моделирование не учитывает электронное поглощение, но описывает магнитное состояние и динамические процессы, как в ЖИГ, так и в Ni. Поэтому его результаты являются еще одним доказательством того, что сильное подавление сигнала в эксперименте следует связывать с влиянием $\mathrm{Ni}$ именно как магнетика.

Следует отметить, что на рис. $5, b$ приведена лишь та часть области моделирования, где колебания намагниченности для структуры В были наиболее ярко выражены. На опущенном участке колебания $M_{z}$ в выбранном масштабе не видны. Отметим также, что микромагнитное моделирование для структуры В демонстрирует наличие коротковолновых возбуждений на фоне длинноволновых колебаний с $\lambda$, близким к свободной пленке. Столь коротковолновые возбуждения не могут эффективно возбуждаться-приниматься МА, использованными в эксперименте, и поэтому далее не обсуждаются. При этом стоит отметить, что их наличие может являться артефактом вследствие упрощения действия МА при моделировании. Возможно также, что возбуждению коротких СВ в данном случае способствует наличие магнитных неоднородностей (границ области моделирования, а также границы микроструктуры $\mathrm{Ni}$ ).

\section{Структуры C, D и E}

В структуре C наличие микроканала, свободного от $\mathrm{Ni}$, обеспечивало эффективное прохождение сигнала к MA 2 в той же полосе частот, что и в структуре A, тогда как АЧХ $S_{31}$ с точностью эксперимента совпадала с результатами для структуры В - см. рис. 2. АЧХ $S_{21}$ характеризовалась повышением затухания сигнала с ростом частоты по сравнению с результатами для структуры А. При этом при $H>400$ Ое в АЧХ наблюдалась серия осцилляций, глубина которых увеличивалась с ростом $H$. Экспериментальная дисперсия, определенная из $S_{21}$, была близка к случаю свободной пленки - см. рис. 3.

Микромагнитное моделирование демонстрировало качественное согласие с экспериментом: ПМСВ под $\mathrm{Ni}$ подавлялась, и наблюдалось прохождение волны лишь в микроканале, свободном от $\mathrm{Ni}$ (рис. 5, c). При этом можно видеть наличие некой структуры у волнового поля по ширине канала, что может быть связано с многомодовым режимом распространения ПМСВ в нем. Видно также, что волновой пучок ПМСВ не ограничивается строго геометрической границей волнового канала, сформированного пленкой Ni. Однако магнитные колебания под $\mathrm{Ni}$ быстро спадают по мере продвижения от границы волнового канала.

Для структуры D характеристика $\left|S_{31}\right|$ была близка к случаю структуры А (рис. 2). При этом АЧХ $S_{21}$ была близка к результатам для структуры В лишь в очень слабых полях. С ростом $H$ прохождение сигнала, отвечающее высокочастотной части $\left|S_{21}\right|$, улучшалось. При $H>800$ Ое в АЧХ появлялась серия осцилляций. 
Экспериментальная дисперсия, определенная из $S_{21}$, с хорошей точностью совпадала с результатами для свободной пленки (рис. 3). Это указывает на то, что большая часть энергии от МА 1 к МА 2 передавалась по свободной пленке, огибая микрополоску Ni. Об этом же свидетельствуют и результаты микромагнитного моделирования - см. рис. 5, $d$. При этом микромагнитное моделирование демонстрирует также небольшое изменение волнового фронта по сравнению со структурой А: вблизи от микрополоски $\mathrm{Ni}$ и под ней волновой фронт выгибается в сторону МА 2, что можно интерпретировать как повышение фазовой скорости ПМСВ в этом месте.

При сравнении АЧХ $S_{21}$, отвечающих структурам С и $\mathrm{D}$, обращает на себя внимание тот факт, что осцилляции в АЧХ для этих двух структур чередуются: минимумы прохождения для структуры С отвечают максимумам прохождения для структуры D и наоборот (см. рис. 2, а также вставку на рис. 3). Анализ положения указанных осцилляций с точки зрения дисперсионной характеристики показывает, что они с хорошей точностью соответствуют укладыванию целого числа полуволн ПМСВ на длине $L_{\mathrm{Ni}}: L_{\mathrm{Ni}}=\lambda \cdot n / 2$, где $n-$ целое число. Для наглядности на вставке на рис. 3 штриховыми и пунктирными линиями отмечены $k$ и $f$ ПМСВ, отвечающие этому условию при нечетных и четных $n$ соответственно. Видно, что для структуры С минимумы прохождения соответствуют нечетным $n$, а максимумы - четным. Для структуры D ситуация обратная. В целом это указывает, что данные осцилляции в АЧХ связаны с установлением резонансов стоячих ПМСВ на длине $L_{\mathrm{Ni}}$, однако причина различия в положении осцилляций для структур C и D пока не ясна.

Для структуры Е область частот, отвечающая высокочастотной (коротковолновой) части спектра ПМСВ, характеризовалась эффективной передачей сигнала к MA 3 и слабой передачей к МА 2 - см. рис. 2. Наиболее ярко это проявлялось в слабых полях подмагничивания. Результаты микромагнитного моделирования (рис. 5,e) качественно согласуются с экспериментом. При этом видно, что волновой пучок, распространяющийся в канале от МА 1 к 3, также как и ПМСВ в структуре А характеризуется направлением фазовой скорости, близким к оси $y$.

\section{6. Заключение}

Таким образом, в работе показано, что в пленке ЖИГ, нагруженной $\mathrm{Ni}$, ПМСВ может испытывать значительное подавление, что в свою очередь может быть использовано для управления волновыми потоками и формирования отдельных волновых пучков в пленке ЖИГ за счет создания на ее поверхности структур из $\mathrm{Ni}$. При этом анизотропность характеристик ПМСВ позволяет обеспечить эффективную передачу сигнала под различными углами к полю подмагничивания. Обнаружен эффект формирования пространственных резонансов на размерах никелевых структур.

\section{Финансирование работы}

Работа выполнена в рамках государственного задания № 0030-2019-0013 „Спинтроника“ при частичной финансовой поддержке Российского фонда фундаментальных исследований (проект № 18-57-00005).

\section{Конфликт интересов}

Авторы заявляют, что у них нет конфликта интересов.

\section{Список литературы}

[1] V.V. Kruglyak, S.O. Demokritov, D. Grundler. J. Phys. D 43, 264001 (2010).

[2] A.A. Serga, A.V. Chumak, B. Hillebrands. J. Phys. D 43, 264002 (2010).

[3] С.А. Никитов, Д.В. Калябин, И.В. Лисенков, А.Н. Славин, Ю.Н. Барабаненков, С.А. Осокин, А.В. Садовников, Е.Н. Бегинин, М.А. Морозова, Ю.П. Шараевский, Ю.А. Филимонов, Ю.В. Хивинцев, С.Л. Высоцкий, В.К. Сахаров, Е.С. Павлов. УФН 185, 1099 (2015).

[4] A. Khitun, M. Bao, K.L. Wang. J. Phys. D 43, 264005 (2010).

[5] M. Balynsky, A. Kozhevnikov, Y. Khivintsev, T. Bhowmick, D. Gutierrez, H. Chiang, G. Dudko, Y. Filimonov, G. Liu, C. Jiang, A.A. Balandin, R. Lake, A. Khitun. J. Appl. Phys. 121, 024504 (2017).

[6] M. Balynskiy, H. Chiang, A. Kozhevnikov, G. Dudko, Y. Filimonov, A.A. Balandin, A. Khitun. AIP Advances 8, 056619 (2018). DOI: 10.1063/1.5007164

[7] A. Kozhevnikov, F. Gertz, G. Dudko, Y. Filimonov, A. Khitun. Appl. Phys. Lett. 106, 142409 (2015).

[8] Y. Khivintsev, M. Ranjbar, D. Gutierrez, H. Chiang, A. Kozhevnikov, Y. Filimonov, A. Khitun. J. Appl. Phys. 120, 123901 (2016).

[9] D. Gutierrez, H. Chiang, T. Bhowmick, A.D. Volodchenkov, M. Ranjbar, G. Liu, C. Jiang, C. Warren, Y. Khivintsev, Y. Filimonov, J. Garay, R. Lake, A.A. Balandin, A. Khitun. J. Magn. Magn. Mater. 428, 348 (2017).

[10] A. Papp, W. Porod, A.I. Csurgay, G. Csaba. Sci. Rep. 7, 9245 (2017).

[11] А.В. Вашковский, К.В. Гречушкин, А.В. Стальмахов, В.А. Тюлюкин. РЭ 31, 838 (1986).

[12] А.В. Вашковский, К.В. Гречушкин, А.В. Стальмахов, В.А. Тюлюкин. РЭ 32, 1176 (1986).

[13] А.В. Вашковский, А.В. Стальмахов, Д.Г. Шахназарян. Изв. вузов. Физика 31, 67 (1988).

[14] R. Gieniusz, P. Gruszecki, M. Krawczyk, U. Guzowska, A. Stognij, A. Maziewski. Sci. Rep. 7, 8771 (2017).

[15] Г.М. Дудко, А.В. Кожевников, В.К. Сахаров, А.В. Стальмахов, Ю.А. Филимонов, Ю.В. Хивинцев. Изв. Саратовского ун-та. Сер.: Физика 18, 92 (2018).

[16] M. Madami, Y. Khivintsev, G. Gubbiotti, G. Dudko, A. Kozhevnikov, V. Sakharov, A. Stal'makhov, A. Khitun, Y. Filimonov. Appl. Phys. Lett. 113, 152403 (2018). 
[17] A.V. Sadovnikov, E.N. Beginin, S.A. Odincov, S.E. Sheshukova, Y.P. Sharaevskii, A.I. Stognij, S.A. Nikitov. Appl.Phys. Lett. 108, 172411 (2016).

[18] V.S. Tkachenko, A.N. Kuchko, M. Dvornik, V.V. Kruglyak. Appl. Phys. Lett., 101, 152402, (2012). DOI: 10.1063/1.475

[19] A. Sadovnikov, C.S. Davies, S.V. Grishin, V.V. Kruglyak, D.V. Romanenko, Y.P. Sharaevskii, S.A. Nikitov. Appl.Phys. Lett. 106, 192406 (2015).

[20] A.V. Sadovnikov, C.S. Davies, V.V. Kruglyak, D.V. Romanenko, S.V. Grishin, E.N. Beginin, Y.P. Sharaevskii, S.A. Nikitov. Phys. Rev. B 96, 060401 (2017).

[21] А.Ю. Анненков, С.В. Герус, С.И. Ковалев. ЖТФ 74, 98 (2004).

[22] А.Г. Веселов, С.Л. Высоцкий, Г.Т. Казаков, А.Г. Сухаревы, Ю.А. Филимонов. РЭ 39, 2067 (1994).

[23] W.S. Ishak. Proc. IEEE 76, 171 (1988).

[24] В.И. Зубков, В.А. Епанечников, В.И. Щеглов. РЭ 52, 192 (2007).

[25] G.A. Vugalter, A.G. Korovin. J. Phys. D 31, 1309 (1998).

[26] Ю.А. Филимонов, Ю.В. Хивинцев. РЭ 47, 1002 (2002).

[27] M.J. Donahue, D.G. Porter. Interagency Report NISTIR 6376, National Institute of Standards and Technology, Gaithersburg, MD (1999).

[28] А.Г. Гуревич, Г.А. Мелков. Магнитные колебания и волны. Физматлит, М. (1994). 464 с.

[29] R.E. Camley, B.V. McGrath, Y. Khivintsev, Z. Celinski, R. Adam, C.M. Schneider, M. Grimsditch. Phys. Rev. B 78, 024425 (2008).

[30] R.W. Damon, J.R. Eshbach. J. Phys. Chem. Solids 19, 308 (1961).

Редактор Е.Ю. Флегонтова 